\title{
Sequence-independent Inhibition of In Vitro Vascular Smooth Muscle Cell Proliferation, Migration, and In Vivo Neointimal Formation by Phosphorothioate Oligodeoxynucleotides
}

\author{
Weizheng Wang, ${ }^{\star \ddagger}$ Hong Jun Chen, ${ }^{\star \ddagger}$ Allan Schwartz, ${ }^{\star \ddagger}$ Paul J. Cannon, ${ }^{\star \ddagger}$ C.A. Stein, ${ }^{\ddagger \S}$ and LeRoy E. Rabbani ${ }^{\star \ddagger}$ \\ $*$ Division of Cardiology, Departments of ${ }^{\ddagger}$ Medicine and ${ }^{\S}$ Pharmacology, Columbia University College of Physicians and Surgeons, \\ New York 10032
}

\begin{abstract}
Phosphorothioate oligodeoxynucleotides (PS oligos) are antisense (sequence-specific) inhibitors of vascular smooth muscle cell (SMC) proliferation when targeted against different genes. Recently an aptameric G-quartet inhibitory effect of PS oligos has been demonstrated. To determine whether PS oligos manifest non-G-quartet, non-sequence-specific effects on human aortic SMC, we examined the effects of S-dC28, a 28-mer phosphorothioate cytidine homopolymer, on SMC proliferation induced by several SMC mitogens. S-dC28 significantly inhibited SMC proliferation induced by $10 \%$ FBS as well as the mitogens PDGF, bFGF, and EGF without cytotoxicity. Moreover, S-dC28 abrogated PDGF-induced in vitro migration in a modified micro-Boyden chamber. Furthermore, $\mathrm{S}-\mathrm{dC} 28$ manifested in vivo antiproliferative effects in the rat carotid balloon injury model. S-dC28 suppressed neointimal cross-sectional area by $73 \%$ and the intima/media area ratio by $59 \%$. Therefore, PS oligos exert potent non-Gquartet, non-sequence-specific effects on in vitro SMC proliferation and migration as well as in vivo neointimal formation. (J. Clin. Invest. 1996. 98:443-450.) Key words: phosphorothioate oligodeoxynucleotides - platelet-derived growth factor • basic fibroblast growth factor - epidermal growth factor $\bullet$ heparin-binding growth factors
\end{abstract}

\section{Introduction}

Restenosis after percutaneous transluminal coronary angioplasty remains a vexing problem, occurring in 30-50\% of patients within 3-6 mo of the procedure (1). Moreover, a plethora of different pharmacological strategies to prevent restenosis has proved to be profoundly disappointing (2). Vascular smooth muscle cells $(\mathrm{SMC})^{1}$ have been implicated in the etiology of restenosis $(3,4)$.

Address correspondence to LeRoy E. Rabbani, M.D., Division of Cardiology, Black Building, Room 807, Columbia University College of Physicians and Surgeons, 630 West 168th Street, New York, NY 10032. Phone: 212-305-5365; FAX: 212-305-3679; E-mail: ler8.columbia.edu

Received for publication 15 December 1995 and accepted in revised form 1 May 1996.

1. Abbreviations used in this paper: $\mathrm{bFGF}$, basic fibroblast growth factor; G-quartet, four consecutive guanosines; LDH, lactate dehydrogenase; PS oligos, phosphorothioate oligonucleotides; S-dC28, a 28-mer phosphorothioate cytidine homopolymer; SMC, smooth muscle cell.

J. Clin. Invest.

(C) The American Society for Clinical Investigation, Inc.

0021-9738/96/07/0443/08 \$2.00

Volume 98, Number 2, July 1996, 443-450
This process is characterized by a phenotypic transition from a contractile to synthetic state and is associated with SMC proliferation and SMC migration from the media to the intima $(3,4)$.

Major emphasis has centered on developing sequence-specific antisense strategies to inhibit SMC proliferation and restenosis. Phosphorothioate oligonucleotides (PS oligos) targeted against various protooncogenes involved in cellular proliferation have been used to inhibit restenosis (5). PS oligos are nuclease resistant, isoelectronic congeners of phosphodiester oligonucleotides that are soluble in aqueous solutions and engage in Watson-Crick base pair hybridization (6). Indeed, several different groups have demonstrated antisense inhibition of in vitro SMC proliferation using oligos complementary to the protooncogenes $c$-myb and $c-m y c$ (4, 7-12). Moreover, PS oligos appear to have induced inhibition of restenosis after balloon injury in both the rat carotid artery and porcine models $(7,8,13)$.

Recently, the underlying mechanism of inhibition of both in vitro SMC proliferation and in vivo neointimal hyperplasia after balloon injury by PS oligos complementary to $c-m y b$ and $c-m y c$ has been challenged. One study suggested that the inhibitory effect of these oligos on SMC proliferation in vitro and in vivo is not the result of a hybridization-dependent antisense mechanism, but is the consequence of an aptamer effect produced by four consecutive guanosine (G-quartet) residues present in both the antisense $c-m y b$ and $c$-myc oligos (14). In another study, rat carotid neointimal hyperplasia after balloon injury was not significantly different with antisense $c-m y b$ and sense $c-m y b$ containing G-quartet sequences (5). In vitro SMC proliferation was inhibited by both sense and antisense $c-m y b$ oligos containing the consecutive G-quartet sequences (5).

Recently, PS oligos have been demonstrated to possess additional properties which may be germane to their SMC antiproliferative effects. Indeed, PS oligos have been demonstrated to bind directly to various heparin-binding growth factors such as basic fibroblast growth factor (bFGF) and platelet-derived growth factor (PDGF) (15). Given the confusion surrounding the mechanism of action of the SMC antiproliferative effects of the PS oligos and the recent demonstration of nonantisense, non-sequence-specific effects of the PS oligos, we hypothesized that the inhibitory effects of the PS oligos on SMC proliferation in vitro and neointimal hyperplasia after balloon injury in vivo are the direct result of non-G-quartet, non-sequence-specific inhibition of growth factors by the PS oligos. Therefore, in this study, we examined the effects of S-dC28, a 28-mer phosphorothioate cytidine homopolymer, which lacks a contiguous G-quartet residue. We demonstrate here for the first time that the PS oligo, S-dC28, exerts potent non-G-quartet, non-sequence-specific inhibitory effects on in vitro human aortic SMC proliferation induced by several mitogens, in vitro human aortic SMC migration, and in vivo neointimal formation after balloon injury in the rat carotid artery model. 


\section{Methods}

Materials. S-dC28 and S-dT28 were synthesized on a DNA synthesizer (model 380B; Applied Biosystems, Foster City, CA) and purified with reverse phase high pressure liquid chromatography as previously described (16). PDGF BB, AA, and AB, bFGF, and epidermal growth factor (EGF) were purchased from Upstate Biotechnology Inc. (Lake Placid, NY); CytoTox $96^{\mathrm{TM}}$ cytotoxicity assay system and CellTiter $96^{\mathrm{TM}}$ AQ proliferation assay kits were purchased from Promega Corp. (Madison, WI). FBS and M199 were purchased from Gibco Laboratories (Grand Island, NY).

Cell culture. Human aortic SMC were obtained from Clonetics Corp. (San Diego, CA) and subcultured using smooth muscle growth medium (Clonetics) containing human EGF $(10 \mathrm{ng} / \mathrm{ml})$, human fibroblast growth factor $(2 \mathrm{ng} / \mathrm{ml})$, dexamethasone $(0.39 \mu \mathrm{g} / \mathrm{ml}), 5 \% \mathrm{FBS}$, gentamycin $(50 \mu \mathrm{g} / \mathrm{ml})$, and amphotericin-B $(50 \mathrm{ng} / \mathrm{ml})$ at $37^{\circ} \mathrm{C}$ in a humidified $95 \%$ air $/ 5 \% \mathrm{CO}_{2}$ atmosphere. The growth medium was changed every other day until confluence was reached. The cells used for experiments were from passages 5-10. Verification of SMC phenotype was performed via positive fluorescent staining for $\alpha$-actin (compared with a known positive control for SMC) and negative staining for Factor VIII antigen. Cell viability was $95 \%$ or greater as determined by trypan blue exclusion at the end of experiments.

$S M C$ proliferation assay. Human aortic SMC were grown to 60$70 \%$ confluence in 12-well tissue culture plates $(22.6 \mathrm{~mm}$ diameter; Costar Corp., Cambridge, MA). SMC were then washed three times with basal M199 and incubated with serum-free medium (M199 supplemented with $0.2 \%$ bovine albumin) for $48 \mathrm{~h}$ to obtain quiescent nondividing cells as previously described (17). Thereafter, cells were incubated with equal volumes of vehicle alone, $10 \%$ FBS alone, or $10 \%$ FBS in combination with various concentrations of S-dC28 for $48 \mathrm{~h}$ at $37^{\circ} \mathrm{C}$ in a humidified $95 \%$ air $/ 5 \% \mathrm{CO}_{2}$ atmosphere. SMC were trypsinized and cell numbers were immediately determined by triplicate counts with a Coulter Counter (model Z1; Coulter Electronics, Beds, United Kingdom). The aforementioned experiments were also conducted using different dimeric forms of PDGF BB, AB, and AA. In additional experiments, SMC were treated with S-dC28 $(20 \mu \mathrm{M})$ either $2 \mathrm{~h}$ before or $2 \mathrm{~h}$ after PDGF $(100 \mathrm{ng} / \mathrm{ml})$. In other experiments, SMC were treated with S-dC28 $(20 \mu \mathrm{M})$ for $2 \mathrm{~h}$, followed by the addition of PDGF (100 ng/ml) after washing with medium three times. In another experiment, SMC were treated with the same doses of S-dC28 and PDGF which had been preincubated in a test tube for $2 \mathrm{~h}$. To examine the effects of S-dC28 on other SMC mitogens, the proliferation experiments were also performed with bFGF and EGF.

$S M C$ lactate dehydrogenase $(L D H)$ release. To determine whether $\mathrm{S}-\mathrm{dC} 28$ reduces cell number by causing direct cytotoxicity, we measured SMC LDH release after a 48-h incubation using the CytoTox 96 ${ }^{\mathrm{TM}}$ cytotoxicity assay system (Promega Corp.), a highly sensitive nonradioactive cytotoxicity assay for human target cells (18). Briefly, SMC were seeded to 96-well tissue culture plates and incubated with serum free medium for $48 \mathrm{~h}$ to obtain quiescent nondividing cells (17). Thereafter, cells were incubated with equal volumes of vehicle alone, PDGF (100 ng/ml) alone, or PDGF (100 ng/ml) in combination with various concentrations of S-dC28 for $48 \mathrm{~h}$ at $37^{\circ} \mathrm{C}$ in a humidified $95 \%$ air $/ 5 \% \mathrm{CO}_{2}$ atmosphere. The experiments were conducted in quadruplicate. LDH release from SMC was measured as previously described (18) and results were expressed as a percentage of maximum release. $\mathrm{LDH}$ release was used instead of ${ }^{51} \mathrm{Cr}$ release for SMC cytotoxicity study because the latter is less useful in prolonged experiments $(48 \mathrm{~h})$ due to high ${ }^{51} \mathrm{Cr}$ spontaneous release. SMC viability with the same treatment and incubation periods was also determined by trypan blue exclusion studies in parallel experiments. Briefly, SMC were trypsinized and stained with $0.2 \%$ trypan blue for $1 \mathrm{~min}$ before counting the cells in a hemocytometer.

Tetrazolium calorimetric proliferation assay. A tetrazolium-based nonradioactive proliferation assay, an alternative to $\left[{ }^{3} \mathrm{H}\right]$ thymidine incorporation, was used to determine SMC proliferation. This assay was shown to correlate with $\left[{ }^{3} \mathrm{H}\right]$ thymidine incorporation in studies of growth factor activity (19). Briefly, SMC were seeded to 96-well tissue culture plates and incubated with serum free medium for $48 \mathrm{~h}$ to obtain quiescent nondividing cells (17). Thereafter, cells were incubated with equal volumes of vehicle alone, PDGF $(100 \mathrm{ng} / \mathrm{ml})$ alone, PDGF $(100 \mathrm{ng} / \mathrm{ml})$ in combination with various concentrations of S-dC2 8 for $48 \mathrm{~h}$ at $37^{\circ} \mathrm{C}$ in a humidified $95 \%$ air $/ 5 \% \mathrm{CO}_{2}$ atmosphere. Tetrazolium reagents were added $4 \mathrm{~h}$ before reading the plates with an ELISA card reader. The experiments were conducted in quadruplicate.

$S M C$ migration. SMC migration activity was assayed in a modified micro-Boyden chamber $(20,21)$ using a polycarbonate filter of $8.0 \mu \mathrm{m}$ (diameter) pore size (Costar Corp.) to divide the upper and lower well chambers. Cultured human aortic SMC were trypsinized and suspended at a concentration of $5 \times 10^{5}$ cells $/ \mathrm{ml}$ in M199 supplemented with $0.2 \%$ bovine albumin. A volume of $1 \mathrm{ml}$ of cell suspension was placed in the upper chamber, and $2 \mathrm{ml}$ of the same medium containing vehicle, PDGF $(50 \mathrm{ng} / \mathrm{ml})$, or PDGF in combination with varying concentrations of S-dC28 was loaded in the lower chamber of the apparatus. After $48 \mathrm{~h}$ of incubation $\left(37^{\circ} \mathrm{C}, 5 \% \mathrm{CO}_{2}\right.$ in air), the cells on the upper and lower sides of the filter were trypsinized and counted using a Coulter Counter. Migration activity was determined by the ratio of cell number of triplicate counts in the upper and lower chambers of the apparatus.

Rat balloon injury studies. Male Sprague-Dawley rats (Charles River Breeding Laboratories, Wilmington, MA), weighing 400-500 grams, were used. All animal procedures were approved by the Columbia University Institutional Animal Care and Use Committee, Health Sciences Division. Each animal was anesthetized with an intraperitoneal injection of $100 \mathrm{mg} / \mathrm{kg}$ ketamine (Ketalar; Parke Davis, Morris Plains, NJ), and $5 \mathrm{mg} / \mathrm{kg}$ of xylazine (Rompum; Mobay Corp., Shawnee, KS). The right carotid artery of each animal was isolated by a midline incision, suspended on ties, and stripped of adventitia as previously described (22). A $2 \mathrm{~F}$ Fogarty catheter was introduced through the external carotid artery of each rat and was advanced to the aortic arch; the balloon was inflated in the common carotid artery to produce moderate resistance to catheter movement and then gradually withdrawn to the entry point. The entire procedure was repeated three times for each animal. Immediately after balloon injury, $150 \mu \mathrm{l}$ of solution of saline, $25 \%$ pluronic gel, or $25 \%$ pluronic gel containing S-dC28 $120 \mu \mathrm{M}$ was applied to the exposed adventitial surface of the carotid artery at the site of balloon injury. The pluronic solutions were prepared as outlined by the manufacturer (BASF Wyandotte Corporation, Wyandotte, MI) and maintained at $4^{\circ} \mathrm{C}$. Prechilled pipettes and tips were used to apply the gel solutions to the common carotid arteries. The treated area constituted about half of the carotid artery and represented the portion that lies within the neck. On contact with tissues at $37^{\circ} \mathrm{C}$, the pluronic solutions gelled instantaneously generating a translucent layer that enveloped the treated region. The wounds were sutured immediately after application of gel, and the rats were returned to the cages. In some experiments, a suture was placed to mark dividing points between treated and untreated carotid segments. Inspection of additional animals revealed that the pluronic gel disappeared over $1-2 \mathrm{~h} .14 \mathrm{~d}$ after balloon injury, the animals were anesthetized with Nembutal and perfused with saline. The carotid arteries were removed, fixed in $4 \%$ formalin, and processed for light microscopy in a standard manner.

Statistics. Data are presented as the mean \pm SD of the independent experiments. Statistical significance was determined by one-way ANOVA and Fisher's PLSD test (StatView 4.01; Brain Power, Inc., Calabasas, CA). For data of treated and untreated segments of carotid arteries, a paired $t$ test (StatView 4.01) was used. A $P$ value of $<0.05$ was considered statistically significant between the means.

\section{Results}

Effects of S-dC28 on SMC proliferation in vitro. We examined the effects of varying concentrations of S-dC28 on SMC prolif- 


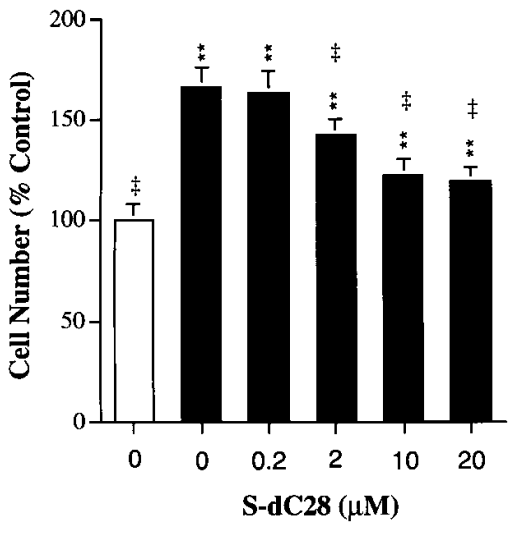

were immediately determined by triplicate counts with a Coulter Counter. Values are mean $\pm \operatorname{SD}(n=6) . * * P<0.01$ relative to control SMC cultured in media alone, ${ }^{\ddagger} P<0.01$ compared with the $10 \%$ FBS alone group. Open bars, control; filled bars, $10 \%$ FBS + S-dC28.

eration induced by $10 \%$ FBS (Fig. 1). SMC number after coincubation with $10 \%$ FBS was significantly increased over the value of the control group. S-dC28 at concentrations of $10 \mu \mathrm{M}$ (66\% inhibition, $P<0.001)$ and $20 \mu \mathrm{M}$ (72\% inhibition, $P<$ $0.001)$ significantly inhibited SMC proliferation induced by $10 \%$ FBS $(n=8)$. The effects of S-dC28 on SMC proliferation induced by human recombinant PDGF BB are depicted in Fig. 2. PDGF significantly increased cell number over the value of the control group $(n=7, P<0.0001)$. S-dC28 at concentrations of $10 \mu \mathrm{M}(57 \%$ inhibition, $P<0.01)$ and $20 \mu \mathrm{M}(90 \%$ inhibition, $P<0.001$ ) significantly inhibited SMC proliferation induced by PDGF $(n=7)$. S-dC28 itself did not affect SMC proliferation in serum-free media. The modulation of the effects of S-dC28 on PDGF-mediated SMC proliferation is presented in Table I. Addition of S-dC28 $2 \mathrm{~h}$ after pretreatment with PDGF reduced SMC proliferation by $60 \%$. S-dC28 pretreatment of SMC $2 \mathrm{~h}$ before the addition of PDGF inhibited SMC proliferation by $79 \%(P<0.01)$. However, when SMC were preincubated with $\mathrm{S}-\mathrm{dC} 28$ for $2 \mathrm{~h}$ and then washed three times with medium, the subsequent addition of PDGF resulted in only a 37\% attenuation in SMC proliferation. S-dC28 and

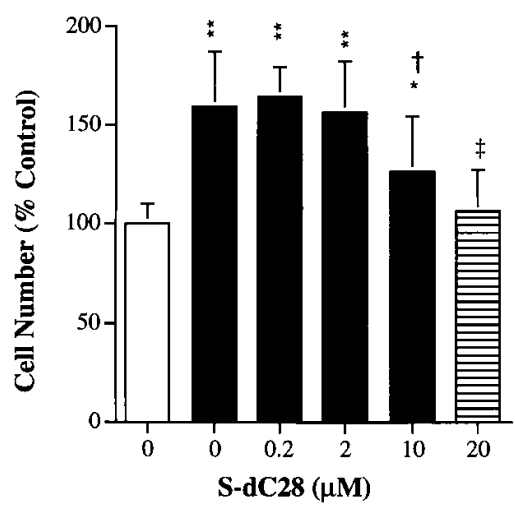

Figure 2. Effects of S-dC28 on human aortic SMC proliferation stimulated by PDGF. Growth-arrested human aortic SMC were incubated with vehicle alone, PDGF (100 ng/ $\mathrm{ml})$ alone, or PDGF $(100 \mathrm{ng} / \mathrm{ml})$ in combination with various doses of S-dC28 for $48 \mathrm{~h}$ at $37^{\circ} \mathrm{C}$ in a humidified $5 \% \mathrm{CO}_{2}$ atmosphere. $\mathrm{SMC}$ were then

trypsinized and cell numbers were immediately determined by triplicate counts with a Coulter Counter. Values are mean $\pm \operatorname{SD}(n=7)$. $* * P<0.01$ relative to control SMC cultured in media alone; ${ }^{\ddagger} P<0.01$ compared with the PDGF alone group. Open bars, control; filled bars, PDGF + S-dC28; striped bars, S-dC28 alone.
Table I. Modulation of the Antiproliferative Effects of $S$-dC28 on PDGF-induced SMC Proliferation

\begin{tabular}{llcc}
\hline & \multicolumn{2}{c}{ Time of addition (h) } & \\
\cline { 2 - 3 } \multicolumn{1}{c}{ Group } & PDGF & S-dC28 & $\begin{array}{c}\text { Percent inhibition of } \\
\text { SMC proliferation }\end{array}$ \\
\hline PDGF & 0 & - & $0 \%$ \\
PDGF + S-dC28 & 0 & 2 & $60 \% *$ \\
S-dC28 + PDGF & 2 & 0 & $79 \% *$ \\
S-dC28 + PDGF & 2 & 0 & $37 \% \S$ \\
PDGF + S-dC28 & 0 & 0 & $63 \% *$ \\
& & & \\
\hline
\end{tabular}

Growth-arrested human aortic SMC were incubated with vehicle alone, PDGF (100 ng/ml) alone, or PDGF (100 ng/ml) in combination with 20 $\mu \mathrm{M}$ of S-dC28 at $37^{\circ} \mathrm{C}$ in a humidified $5 \% \mathrm{CO}_{2}$ atmosphere for $48 \mathrm{~h}$. $\mathrm{SMC}$ were then trypsinized and cell numbers were immidiately determined by triplicate counts with a Coulter Counter. ${ }^{\ddagger}$ SMC pretreated with $\mathrm{S}$-dC28 for $2 \mathrm{~h}$, followed by three washes with medium and subsequent addition of PDGF. "SMC incubated with PDGF and S-dC28 which had been prevously mixed together in a test tube for $2 \mathrm{~h}$. Values are mean $\pm \mathrm{SD}(n=6),{ }^{\S} P<0.05,{ }^{*} P<0.01$ relative to the PDGF group.

PDGF preincubated in a test tube for $2 \mathrm{~h}$ before addition to SMC reduced SMC proliferation by $63 \%$.

To determine whether S-dC28's inhibition of SMC proliferation is specific to PDGF, we also conducted the experiments using other SMC mitogens. The effects of S-dC28 on bFGFinduced and EGF-induced SMC proliferation are shown in Table II. bFGF (10 ng/ml) significantly increased SMC number. SMC number after coincubation with bFGF (10 ng/ml) and S-dC28 at concentrations of $10 \mu \mathrm{M}(67 \%$ inhibition, $P<$ $0.001)$ and $20 \mu \mathrm{M}(76 \%$ inhibition, $P<0.001)$ was significantly

Table II. Effects of S-dC28 on bFGF- and EGF-induced SMC Proliferation

\begin{tabular}{lcccc}
\hline \multicolumn{1}{c}{ Group } & bFGF & EGF & S-dC28 & $\begin{array}{c}\text { Percent inhibition of } \\
\text { SMC proliferation }\end{array}$ \\
\hline & $n g / m l$ & $n g / m l$ & $\mu M$ & \\
bFGF & 10 & - & - & $0 \%$ \\
bFGF + S-dC28 $2 \mu \mathrm{M}$ & 10 & - & 2 & $15 \%$ \\
$\mathrm{bFGF}+\mathrm{S}-\mathrm{dC} 2810 \mu \mathrm{M}$ & 10 & - & 10 & $67 \% *$ \\
$\mathrm{bFGF}+\mathrm{S}-\mathrm{dC} 2820 \mu \mathrm{M}$ & 10 & - & 20 & $76 \% *$ \\
& - & 10 & - & $0 \%$ \\
$\mathrm{EGF}$ & - & 10 & 2 & $13 \%$ \\
$\mathrm{EGF}+\mathrm{S}-\mathrm{dC} 282 \mu \mathrm{M}$ & - & 10 & 10 & $63 \% *$ \\
$\mathrm{EGF}+\mathrm{S}-\mathrm{dC} 2810 \mu \mathrm{M}$ & - & 10 & 20 & $77 \% *$ \\
$\mathrm{EGF}+\mathrm{S}-\mathrm{dC} 2820 \mu \mathrm{M}$ & - & & & \\
\hline
\end{tabular}

Growth-arrested human aortic SMC were incubated with vehicle alone, bFGF (10 ng/ml) alone, of bFGF (10 ng/ml) in combination with various doses of S-dC28 for $48 \mathrm{~h}$ at $37^{\circ} \mathrm{C}$ in a humidified $5 \% \mathrm{CO}_{2}$ atmosphere. In other experiments, growth-arrested human aortic SMC were incubated with EGF $(10 \mathrm{ng} / \mathrm{ml})$ alone or EGF $(10 \mathrm{ng} / \mathrm{ml})$ in combination with various doses of $\mathrm{S}-\mathrm{dC} 28$ for $48 \mathrm{~h}$ at the same conditions. SMC were then trypsinized and cell numbers were immediately determined by triplicate counts with a Coulter Counter. Values are expressed as the percentage of inhibition of bFGF- and EGF-induced SMC proliferation. ${ }^{*} P<0.01$ relative to the bFGF alone group or the EGF alone group. 


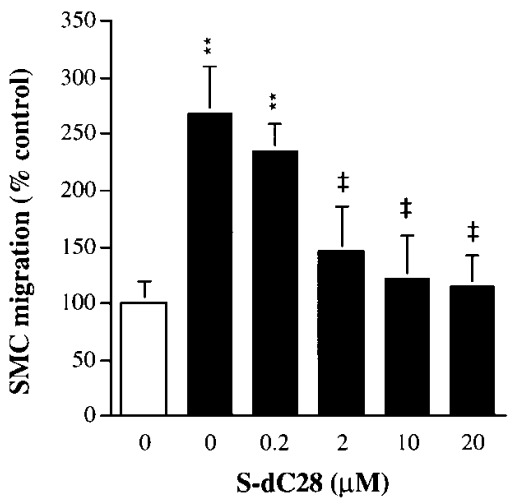

Figure 3. Effects of S-dC28 on SMC migration. SMC migration studies were performed using a modified microBoyden chamber. Human aortic SMC $(5 \times$ $\left.10^{5} / \mathrm{ml}\right)$ were added into the upper chamber of the transwell, while vehicle alone, PDGF (100 $\mathrm{ng} / \mathrm{ml}$ ) alone, or PDGF $(100 \mathrm{ng} / \mathrm{ml})$ in combination with various doses of S-dC28 was added to the lower chamber. SMC migration was determined using the Coulter Counter after a 48 -h incubation at $37^{\circ} \mathrm{C}$ in a humidified $5 \% \mathrm{CO}_{2}$ atmosphere. Migration activity was determined by the ratio of cell number of triplicate counts in the upper and lower chambers of the apparatus. Values are mean $\pm \mathrm{SD}(n=6) . * * P<0.01$ relative to control SMC cultured in media alone; ${ }^{\ddagger} P<0.01$ compared with the PDGF alone group. Open bars, control; filled bars, PDGF + S-dC28.

attenuated compared with the values of the bFGF alone group $(n=5)$. SMC number after coincubation with $10 \mathrm{ng} / \mathrm{ml}$ of EGF was significantly increased. S-dC28 at concentrations of $10 \mu \mathrm{M}$ (63\% inhibition, $P<0.01)$ and $20 \mu \mathrm{M}$ (77\% inhibition, $P<0.001)$ significantly inhibited SMC proliferation induced by EGF $(n=4)$. To test whether PS oligos other than S-dC28 have similar effects on SMC proliferation, the effect of S-dT28 on SMC proliferation induced by PDGF was studied. Similar inhibition of SMC proliferation was demonstrated (data not shown).

Effects of $S$ - $d C 28$ on the tetrazolium-based proliferation assay. PDGF significantly increased SMC proliferation in the tetrazolium-based proliferation assay over the value of the control group $(n=7, P<0.001)$. S-dC28 at concentrations of $20 \mu \mathrm{M}(99 \%$ inhibition, $P<0.01)$ and $10 \mu \mathrm{M}(52 \%$ inhibition, $P<0.001)$ significantly inhibited SMC proliferation induced by PDGF $(n=5)$. These results, which reflect DNA synthesis in SMC, were well correlated to the direct cell counts.

Effects of $S$-dC28 on LDH release. To determine whether S-dC28's effects on SMC proliferation are a result of its cytotoxic effects on SMC, we measured SMC LDH release after a 48 -h incubation with various dosages of S-dC28. S-dC28 concentrations from 0.02 to $20 \mu \mathrm{M}$ did not alter SMC LDH release ( $<7 \%$ of maximum release in all groups) $(n=6)$. In parallel experiments, we also found that $\mathrm{S}-\mathrm{dC} 28$ did not alter
SMC viability ( $\geq 95 \%$ in all groups) at the end of experiments as determined by trypan blue exclusion $(n=6)$.

Effect of $S-d C 28$ on in vitro $S M C$ migration. SMC migration studies using a modified micro-Boyden chamber are shown in Fig. 3. PDGF-induced SMC migration from the upper chamber to the lower chamber was significantly greater $(268 \pm 42 \%)$ than the value of SMC migration in the control group. S-dC28 at concentrations of $2 \mu \mathrm{M}(P<0.011), 10 \mu \mathrm{M}(P<0.003)$, and $20 \mu \mathrm{M}(P<0.002)$ significantly inhibited SMC migration induced by PDGF in a dose-dependent fashion $(n=4)$.

Effects of $S$-dC28 on neointima formation of rat carotid arteries after balloon angioplasty injury. To examine whether S-dC28 also suppresses SMC proliferation and migration in vivo, we used the rat right carotid artery balloon angioplasty model, which denudes endothelium and induces a highly reproducible neointimal proliferation and migration of SMC over the entire length of the injured blood vessel $(23,24)$. We have determined the effects of S-dC28 on intimal SMC accumulation $2 \mathrm{wk}$ after angioplasty in 20 rats which were treated with pluronic gel containing S-dC28 $(n=9)$, pluronic gel alone $(n=7)$, or saline $(n=4)$. The PS oligos used in this study were well tolerated by the rats; none of the PS oligo-treated rats developed side effects or died before time of killing. All of the experiments described were performed in a blinded fashion. Morphological examination revealed that minimal neointimal accumulation developed in the S-dC28-treated group, whereas extensive neointimal accumulation was observed in the pluronic gel alone and saline groups (Fig. 4). The measurements of arterial segments demonstrated that S-dC28 $(n=9)$ suppressed the neointimal SMC accumulation (cross-sectional area) compared with the values in the saline group $(n=4, P<$ $0.004)$ and the pluronic gel group $(n=7, P<0.0001$; Fig. $5 a)$. Furthermore, the intima/media area ratio was significantly reduced by $\mathrm{S}-\mathrm{dC} 28$ treatment over the values of the saline group ( $n=4, P<0.0006)$ and the pluronic gel group $(n=7, P<$ $0.0001)$, indicating minimal effects on medial SMC growth (Fig. $5 b$ ).

We also examined the rat carotid arteries to evaluate the longitudinal extent of suppression of neointimal SMC accumulation after balloon injury. Fig. 6 shows a representative transitional region of a treated zone (in the neck) and an untreated zone (in the chest) of the same carotid artery which had been subjected to balloon injury. Measurements of the treated and the untreated segments of the same carotid arteries documented that S-dC28 significantly suppressed the neointimal SMC accumulation in the treated zones (intimal area $P<$
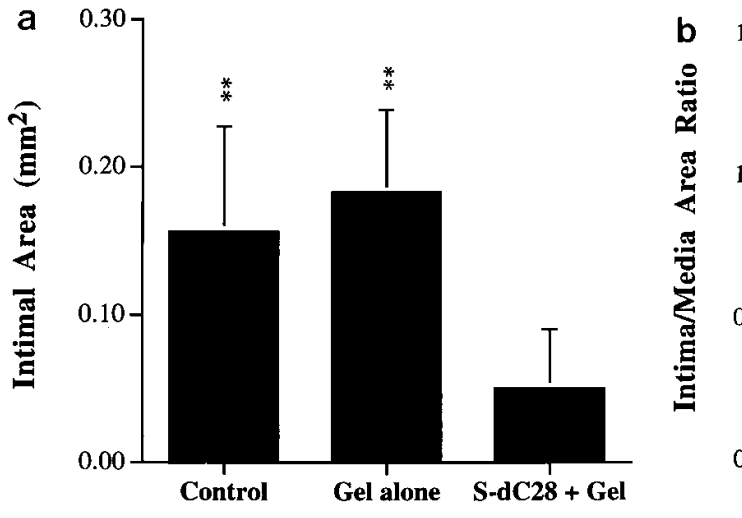

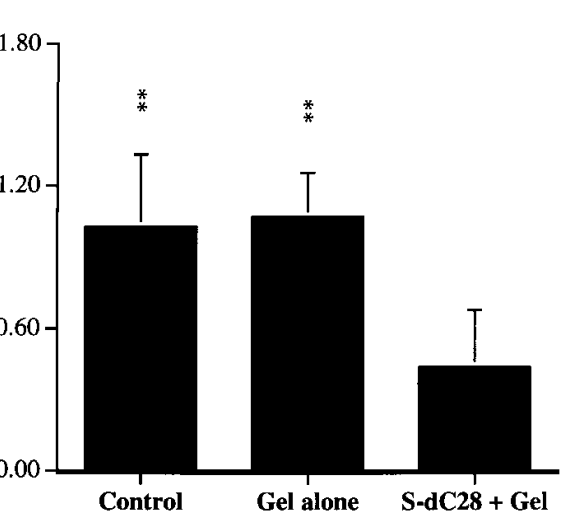

Figure 5. Effects of S-dC28 on rat common carotid artery neointimal cross-sectional area (a) as well as the intima/media ratio $(b)$. Rat carotid arteries after balloon injury were treated with saline (control group, $n=$ 4), pluronic gel alone (gel group, $n=7$ ), and pluronic gel containing S-dC28 (S-dC28 + gel group, $n=9)$ for $14 \mathrm{~d}$. All the measurements were performed in a blinded fashion. Values are mean $\pm \mathrm{SD} * * P<0.01$ relative to the $\mathrm{S}-\mathrm{dC} 28+$ gel group. 

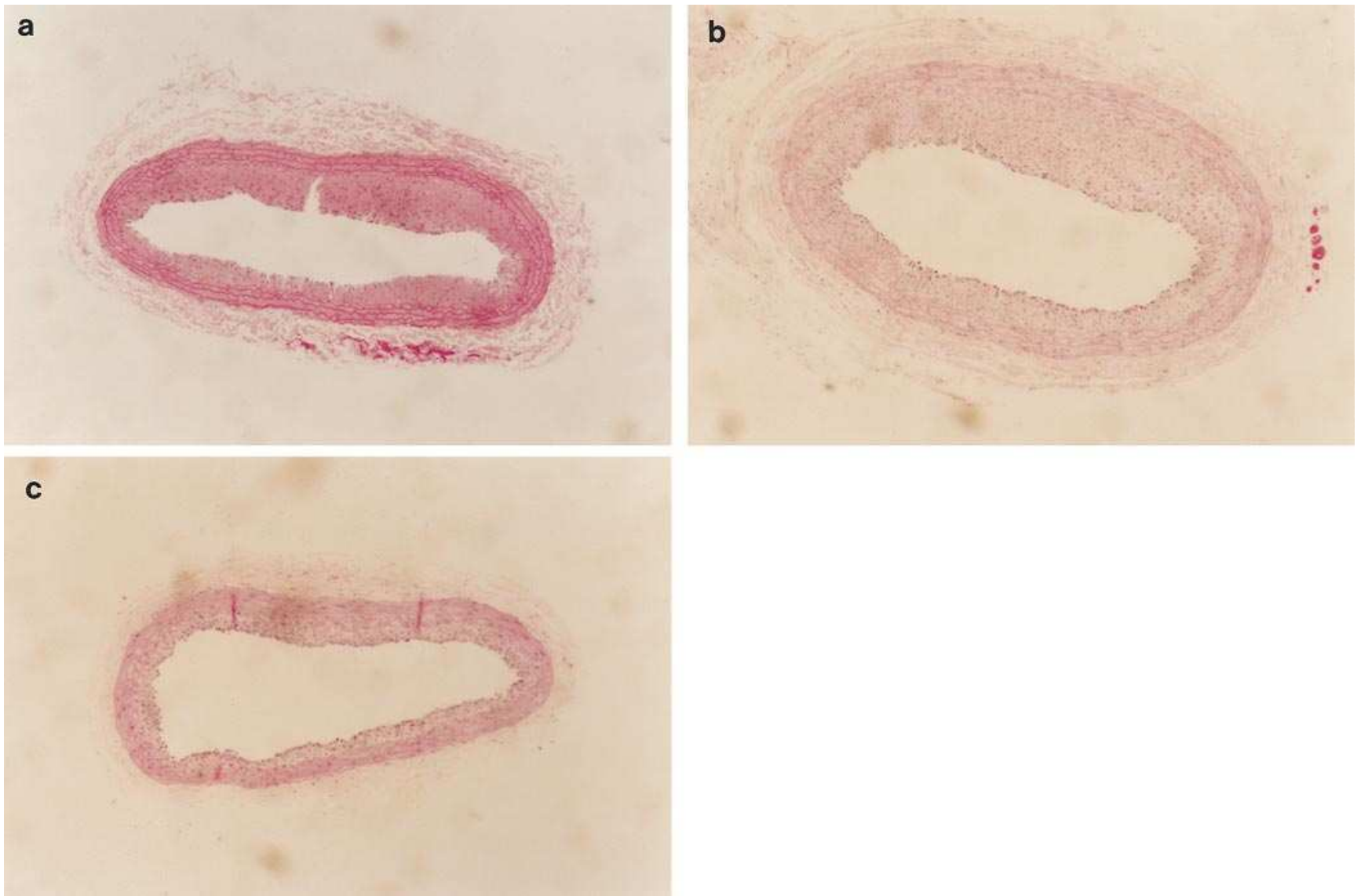

Figure 4. Effects of S-dC28 on neointimal formation in rat carotid arteries subjected to balloon angioplasty. Representative cross-sections are shown from the carotid artery of an untreated rat $(a)$, a rat treated with pluronic gel alone $(b)$, and a rat treated with pluronic gel containing S-dC28 (c) (Nikon, $\times 80)$. The tissue specimens were hematoxylin and eosin stained for microscopy in a standard manner.

0.017 ; intima/media area ratio $P<0.017)$ compared with the values in the untreated zones $(n=5)$ (Fig. 7).

\section{Discussion}

In this study, we demonstrate that PS oligos manifest nonG-quartet, non-sequence-specific antiproliferative effects on

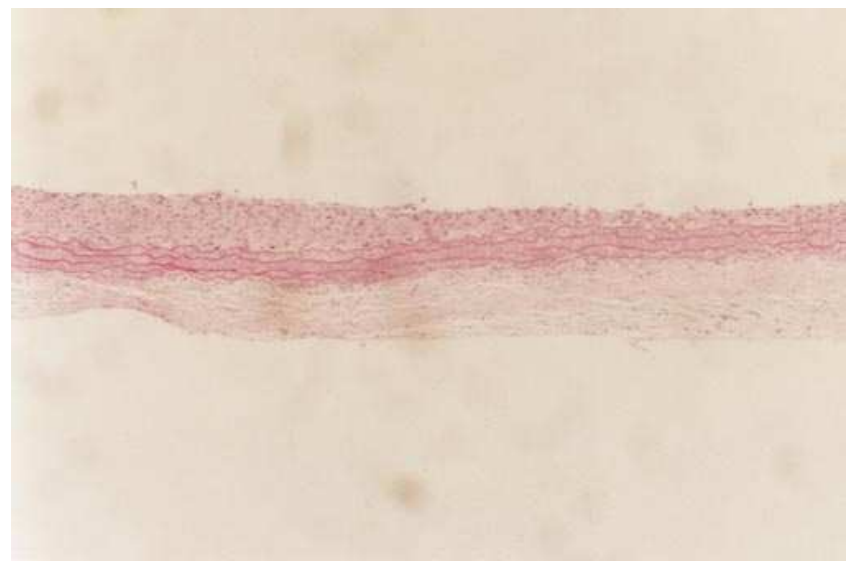

Figure 6. Spatial distribution of the antiproliferative effects of $\mathrm{S}-\mathrm{dC} 28$. The longitudinal section of the transitional area between the treated and untreated zones (Nikon, $\times 80)$ is depicted.
SMC in vitro and in vivo. S-dC28 inhibited human aortic SMC proliferation in vitro induced by $10 \%$ FBS as well as by the mitogens PDGF, bFGF, and EGF. S-dC28 also inhibited PDGF induction of human aortic SMC proliferation measured by a nonradioactive proliferation assay which correlates with DNA synthesis (19). S-dC28 did not reduce cell number by causing direct cell cytotoxicity as evinced by measurements of the LDH release and trypan blue exclusion. In addition, S-dC28

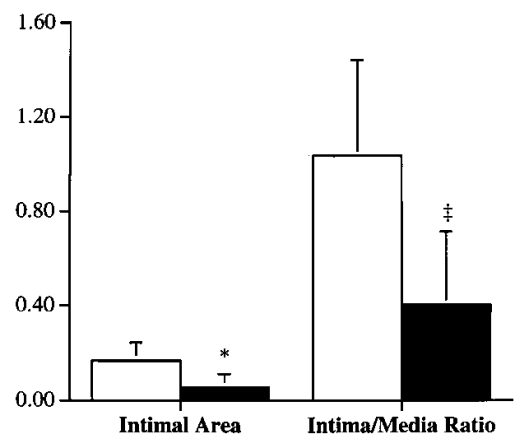

Figure 7. Effects of S-dC28 on rat common carotid artery intimal cross-sectional area as well as the intima/media ratio in treated (filled bars) and untreated segments (open bars). Rat carotid arteries after balloon injury were treated with pluronic gel plus S-dC28 (S-dC28 + gel group, $n=9$ ) for $14 \mathrm{~d}$. Treated segments represented the neck portion of common carotid arteries while untreated segments represented the chest portion of the same arteries which had no S-dC28 treatment. All the measurements were performed in a blinded fashion. Values are mean \pm SD. ${ }^{*} P<0.05,{ }^{\ddagger} P<0.01$ relative to the $\mathrm{S}-\mathrm{dC} 28+$ gel group. 
abrogated PDGF-induced SMC migration in vitro in a modified micro-Boyden chamber. Furthermore, S-dC28 manifested antiproliferative effects in the rat carotid balloon injury model in vivo. S-dC28 suppressed neointimal SMC cross-sectional area measured at $2 \mathrm{wk}$ after balloon injury by $73 \%$ and the intima/media area ratio by $59 \%$. This sequence-independent inhibition manifested by PS oligos that we observed on SMC proliferation and migration in vitro and neointimal hyperplasia in vivo after balloon injury is most likely a reflection of PS oligo inhibition of the activity of SMC growth factors through PS oligo binding to growth factors and to cellular and extracellular proteins at multiple sites.

In this study, S-dC28 inhibited in vitro SMC proliferation induced by $10 \%$ FBS as well as by PDGF, bFGF, and EGF. Most of the large effect observed with $10 \%$ FBS may have represented inhibition by $\mathrm{S}-\mathrm{dC} 28$ of PDGF present in FBS. PDGF is the major mitogen present in serum that stimulates SMC DNA synthesis and cell growth $(25,26)$. Moreover, in our study, S-dC28 also inhibited PDGF-induced SMC migration in vitro in a modified micro-Boyden chamber.

PS oligos have been reported to bind directly to heparinbinding growth factors such as bFGF, acidic fibroblast growth factor, vascular endothelial cell growth factor, and fibroblast growth factor 4 (15). PS oligo binding to heparin-binding growth factors is similar to that of other polyanions such as suramin and pentosan polysulfate (15). Periadventitially administered sulfated $\beta$-cyclodextrin polymer, which tightly binds heparin-binding growth factors, induces sustained inhibition of intimal thickening in vivo (27). Using gel mobility assays, it has also been demonstrated that S-dC28 binds directly to PDGF and bFGF but not to EGF (15). Direct binding of S-dC28 to PDGF may account for some of the reduced activity of PDGF to stimulate SMC proliferation when it was preincubated with $\mathrm{S}-\mathrm{dC} 28$ for $2 \mathrm{~h}$ before treatment of the cells (Table I). S-dC28 may also inhibit the growth-promoting activity of SMC mitogens by binding to their receptors on cells. In our study, the partial reversal by washing of the inhibition of PDGF-induced SMC proliferation by S-dC28 that had been preincubated with the cells is consistent with an inhibitory effect of S-dC28 on cell surfaces (Table I).

Previous studies have shown that S-dC28 is taken up by human cells in vitro both by fluid phase pinocytosis and adsorptive endocytosis (28). Internalized S-dC28 may bind to cellular proteins in both the cytoplasm and nucleus (28). Indeed, in our study, maximal inhibition of SMC proliferation was obtained by preincubation of SMC with S-dC28 for $2 \mathrm{~h}$ before the addition of PDGF. Therefore, in addition to its binding to PDGF extracellularly or its inactivation of PDGF on the cell surface, it is possible that $\mathrm{S}-\mathrm{dC} 28$ inactivates signal transduction mechanisms within the cell cytoplasm or nucleus.

Inhibition of the actions of both PDGF and bFGF by direct binding to PS oligos could explain our findings of the in vivo antiproliferative effects of the PS oligos. Indeed, PDGF is also a potent SMC mitogen which has been implicated in SMC migration $(20,29)$. An intravenous infusion of PDGF-BB for $7 \mathrm{~d}$ after rat balloon injury resulted in a 15-fold increase in the neointimal lesion area, principally by increasing SMC migration (20). bFGF is an SMC mitogen (30), and PDGF induction of bFGF has been postulated to play an important role in SMC migration (31). However, it is interesting to note that although S-dC28 binds directly to PDGF and bFGF but not to EGF, it inhibited SMC mitogenesis induced by all three mitogens. De- spite the disparate PS oligo binding properties, PS oligo inhibition of all three mitogens suggests a common pathway of inhibition, possibly at the level of cell surface receptors or via inhibition of an intracellular signal transduction pathway.

Balloon injury-induced release of endogenous bFGF stored in the extracellular matrix has been implicated in the early progress of vascular lesions in the rat carotid artery (30). The potent in vivo antiproliferative effects of S-dC28 may reflect the importance of bFGF in early vascular lesion formation after balloon injury in the rat carotid model. Systemically administered bFGF increased rat vascular SMC proliferation in arteries denuded with a balloon catheter by $54.8 \%$ compared with $11.5 \%$ in controls (30). Administration of a neutralizing antibody directed against bFGF significantly decreased SMC proliferation but not intimal lesion size (32). S-dC28's pleiotropic inhibitory effects and its ability to bind to not only bFGF but also PDGF as well suggest that it may be a very effective and potent antiproliferative agent.

PS oligos have also been demonstrated to modulate the adhesion of vascular cells to elements of the extracellular matrix. PS oligos have been reported to bind to laminin and fibronectin of the extracellular matrix of NIH 3T3 cells (33). PS oligos also inhibited the binding of laminin to bovine brain sulfatide (33). Moreover, PS oligos, in a non-sequence-specific manner, inhibit the binding of fluoresceinated fibronectin to its cell surface receptor in phorbol-12, 13-myristate acetate-treated Jurkat cells (33). The interaction of PS oligos with extracellular matrix proteins of SMC may modulate SMC adhesion and effect an SMC phenotypic change from the synthetic state to the contractile state.

Oligonucleotides manifest other non-sequence-specific pharmacological effects (34) which must be considered in the interpretation of our results. Indeed, one of these is their ability to induce $\gamma$-interferon synthesis by several cell types $(5,34,35)$. $\gamma$-Interferon is a potent inhibitor of in vitro vascular SMC proliferation and the expression of the differentiation-specific $\alpha$-smooth muscle actin antigen in tissue culture $(36,37)$. $\gamma$-Interferon also inhibits in vivo formation of arterial proliferative lesions after rat carotid balloon injury (38). It is a pleiotropic inhibitor of SMC migration induced by PDGF and other promigratory cytokines (39).

S-dC28 manifested potent in vivo antiproliferative effects in our rat carotid injury model. When delivered in pluronic gel, it produced a $73 \%$ decrease in neointimal, cross-sectional area and a $59 \%$ decrease in the intimal $/$ media area ratio. The magnitude of these effects of $\mathrm{S}-\mathrm{dC} 28$ is similar to that observed with other antisense oligonucleotides. Sequence-specific antisense oligonucleotides targeted against $c$ - $m y b$ effected a $95 \%$ reduction in the intimal/media ratio in the study of rat carotid balloon injury of Simons et al. (13) and a reduction of $65 \%$ in the study of Bennett et al. (7) which used a $c-m y c$ antisense oligonucleotide. However, in a more recent study using a rat carotid balloon injury model, treatment with an antisense oligo targeting $c-m y b$ produced no significant reduction in intimal proliferation in vivo (5). Antisense oligonucleotides targeted against cyclin protooncogenes $c d c 2$ and $c d k 2$ and against proliferating-cell nuclear antigen by several groups have also achieved significant (47-95\%) reductions in neointimal hyperplasia in the rat carotid injury model (40-43). However, the present study is the first investigation to demonstrate a significant in vivo effect to reduce neointimal hyperplasia of a PS oligo that was not sequence specific. 
There have been two previous reports that non-sequencespecific PS oligos could inhibit SMC proliferation in vitro (5, 14). In both studies the non-sequence-dependent PS oligos contained constrained multiple consecutive G-quartets $(5,14)$. The authors attributed the antiproliferative effects to aptameric inhibitory effects in some way dependent upon the presence of the G-quartets. The data of the present study of S-dC28 confirm that a non-sequence-dependent PS oligo can inhibit SMC proliferation and also show that it inhibits SMC migration in vitro. However, this study demonstrates that the inhibition of SMC proliferation induced by FBS, PDGF, bFGF, or EGF can be achieved by a PS oligo that lacks G-quartets and is not sequence specific. The extent to which the antisense, nonsequence-specific (non-G-quartet), or G-quartet-dependent inhibitory effects of the PS oligos is observed may depend on various variables such as PS oligo sequence, length, and concentration.

In summary, we have demonstrated that PS oligos exert non-G-quartet, non-sequence-specific inhibition of in vitro SMC proliferation induced by several mitogens, in vitro SMC migration, and in vivo neointimal hyperplasia after balloon injury in the rat carotid artery model. The sequence-independent inhibition is most likely the result of avid binding of PS oligos to a variety of cellular as well as extracellular proteins, including PDGF and heparin-binding growth factors and proteins. Taken together, both the sequence-independent and -dependent properties of the PS oligos need to be considered in the design of optimal PS oligos for the prevention of angioplasty restenosis.

\section{Acknowledgments}

This work was supported by National Institutes of Health (NIH), National Heart, Lung, and Blood Institute Physician-Scientist Award (K11 HLO2578) (L.E. Rabbani), the Milstein Family Foundation (L.E. Rabbani), NIH grant HL21006 (P.J. Cannon), and is partly supported by NIH, National Cancer Institute grant 60639 (C.A. Stein). C.A. Stein is an Irving Scholar of the Columbia University College of Physicians and Surgeons.

\section{References}

1. Nobuyoshi, M., T. Kimura, H.S. Nosaka, S. Moika, K. Ueno, H. Yokui, N. Hamasaki, H. Horiuchi, and H. Ohishi. 1988. Restenosis after successful percutaneous transluminal coronary angioplasty: serial angiographic follow-up of 299 patients. J. Am. Coll. Cardiol. 12:616-623.

2. Popma, J.J., R.M. Califf, and E.J. Topol. 1991. Clinical trials of restenosis after coronary angioplasty. Circulation. 84:1426-1436.

3. Liu, M.W., G.S. Roubin, and S.B. King III. 1989. Restenosis after coronary angioplasty: potential biologic determinants and role of intimal hyperplasia. Circulation. 79:1374-1387.

4. Shi, Y., H.G. Hutchinson, D.J. Hall, and A. Zalewski. 1993. Downregulation of $c$-myc expression by antisense oligonucleotides inhibits proliferation of human smooth muscle cells. Circulation. 88:1190-1195.

5. Villa, A.E., L.A. Guzman, E.J. Poptic, V. Labhasetwar, S. D'Souza, C.L. Farrell, E.F. Plow, R.J. Levy, P.E. DiCorleto, and E.J. Topol. 1995. Effects of antisense $c-m y b$ oligonucleotides on vascular smooth muscle cell proliferation and response to vessel wall injury. Circ. Res. 76:505-513.

6. Stein, C.A., and Y.-C. Cheng. 1993. Antisense oligonucleotides as therapeutic agents-Is the bullet really magical? Science (Wash. DC). 261:1004-1012.

7. Bennett, M.R., S. Anglin, J.R. McEwan, R. Jagoe, A.C. Newby, and G.I. Evan. 1993. Inhibition of vascular smooth muscle cell proliferation in vitro and in vivo by $c-m y c$ antisense oligonucleotides. J. Clin. Invest. 93:820-828.

8. Shi, Y., A. Fard, A. Galeo, H.G. Hutchinson, P. Vernani, G.R. Dodge, D.J. Hall, F. Shaheen, and A. Zalewski. 1994. Transcatheter delivery of $c$-myc antisense oligomers reduces neointimal formation in a porcine model of coronary artery balloon injury. Circulation. 90:944-951.

9. Brown, K.E., M.S. Kindy, and G.E. Sonenshein. 1992. Expression of the c-myc protooncogene in bovine vascular smooth muscle cells. J. Biol. Chem.
267:4625-4630.

10. Simons, M., and R.D. Rosenberg. 1992. Antisense nonmuscle myosin heavy chain and $c-m y b$ oligonucleotides suppress smooth muscle cell proliferation in vitro. Circ. Res. 70:835-843.

11. Ebbecke, M., C. Unterberg, A. Buchwald, S. Stohr, and V. Wiegand. 1992. Antiproliferative effects of a $c-m y c$ antisense oligonucleotide on human arterial smooth muscle cells. Basic Res. Cardiol. 87:585-591.

12. Biro, S., Y.M. Fu, Z.X. Yu, and S.E. Epstein. 1993. Inhibitory effects of antisense oligonucleotides targeting $c-m y c$ mRNA on smooth muscle cell proliferation and migration. Proc. Natl. Acad. Sci. USA. 90:654-658.

13. Simons, M., E.R. Edelman, J.-L. DeKeyser, R. Langer, and R.D. Rosenberg. 1992. Antisense $c-m y b$ oligonucleotides inhibit intimal arterial smooth muscle cell accumulation in vivo. Nature (Lond.). 359:67-70.

14. Burgess, T.L., E.F. Fisher, S.L. Ross, J.V. Bready, Y.-X. Qian, L.A Bayewitch, A.M. Cohen, C.J. Herrera, S.S.-F. Hu, T.B. Kramer, et al. 1995. The antiproliferative activity of $c-m y b$ and $c-m y c$ antisense oligonucleotides in smooth muscle cells is caused by a nonantisense mechanism. Proc. Natl. Acad. Sci. USA. 92:4051-4055.

15. Guvakova, M.A., L.A. Yakubov, I. Vlodavsky, J.L. Tonkinson, and C.A. Stein. 1995. Phosphorothioate oligodeoxynucleotides bind to basic fibroblast growth factor, inhibit its binding to cell surface receptors, and remove it from low affinity binding sites on extracellular matrix. J. Biol. Chem. 270:26202627.

16. Stein, C.A., C. Subasinghe, K. Shinozuka, and J. Cohen. 1988. Physiochemical properties of phosphorothioate oligodeoxynucleotides. Nucleic Acids Res. 16:3209-3221.

17. Yang, Z., G. Noll, and T.F. Luscher. 1993. Calcium antagonists differently inhibit proliferation of human coronary smooth muscle cells in response to pulsatile stretch and platelet-derived growth factor. Circulation. 88:832-836.

18. Franke, L., and T. Portmann. 1994. A highly sensitive non-radioactive cytotoxicity assay for human target cells. J. Immunol. Methods. 171:259-262.

19. Tada, H., O. Shiho, K. Kuroshima, M. Koyamam, and K. Tsukamotok. 1986. An improved colorimetric assay for interleukin-2. J. Immunol. Methods. 93:157-165.

20. Ferns, G.A.A., E.W. Raines, K.H. Sprugel, A.S. Montani, M.A. Reidy, and R. Ross. 1991. Inhibition of neointimal smooth muscle accumulation after angioplasty by an antibody to PDGF. Science (Wash. DC). 253:1129-1132.

21. Jackson, C.L., and M.A. Reidy. 1992. The role of plasminogen activation in smooth muscle cell migration after arterial injury. Ann. NY Acad. Sci. 667:141-150.

22. Edelman, E.R., M.A. Nugent, L.T. Smith, and M.T. Karnovsky. 1992. Basic fibroblast growth factor enhances the coupling of intimal hyperplasia and proliferation of vasa vasorum injured arteries. J. Clin. Invest. 89:465-473.

23. Clowes, A.W., M.A. Reidy, and M.M. Clowes. 1983. Kinetics of cellular proliferation after arterial injury. I. Smooth muscle growth in the absence of endothelium. Lab. Invest. 49:327-333.

24. Edelman, E.R., D.H. Adams, and M.S. Karnovsky. 1990. Effect of controlled adventitial heparin delivery on smooth muscle cell proliferation following endothelial injury. Proc. Natl. Acad. Sci. USA. 87:3773-3777.

25. Ross, R., J. Glomset, B. Kariya, and L. Harker. 1974. A platelet-dependent serum factor that stimulates the proliferation of arterial smooth muscle cells in vitro. Proc. Natl. Acad. Sci. USA. 71:1207-1210.

26. Raines, E.W., and R. Ross. 1982. Platelet-derived growth factor. I. High yield purification and evidence for multiple forms. J. Biol. Chem. 257:51545160 .

27. Bachinsky, W.B., E.S. Barnathan, H. Liu, S.S. Okada, A. Kuo, P.N. Raghunath, M. Muttreja, R.J. Caron, J.E. Tomaszewski, P.B. Weisz, and M.A Golden. 1995. Sustained inhibition of intimal thickening: In vitro and in vivo effects of polymeric $\beta$-cyclodextrin sulfate. J. Clin. Invest. 96:2583-2592.

28. Gao, W.-Y., C. Storm, W. Egan, and Y.-C. Cheng. 1992. Cellular pharmacology of phosphorothioate homooligodeoxynucleotides in human cells. Mol. Pharmacol. 43:45-50.

29. Jackson, C.L., E.W. Raines, R. Ross, and M.A. Reidy. 1993. Role of endogenous platelet-derived growth factor in arterial smooth muscle cell migration after balloon catheter injury. Arterioscler. Thromb. 13:1218-1226.

30. Lindner, V., D.A. Lappi, A. Baird, R.A. Majack, and M.A. Reidy. 1991 Role of basic fibroblast growth factor in vascular lesion formation. Circ. Res. 68 : 106-113.

31. Soto, Y., R. Hamanaka, J. Ono, M. Kuwano, D.B. Rifkin, and R. Takaki. 1991. The stimulatory effect of PDGF on vascular smooth muscle cell migration is mediated by the induction of endogenous basic FGF. Biochem. Biophys. Res. Commun. 174:1260-1266.

32. Lindner, V., and M.A. Reidy. 1991. Proliferation of smooth muscle cells after vascular injury is inhibited by an antibody against basic fibroblast growth factor. Proc. Natl. Acad. Sci. USA. 88:3739-3743.

33. Khaled, Z., L. Benimetskaya, R. Zeltser, T. Khan, H.W. Sharma, R. Narayanan, and C.A. Stein. 1996. Multiple mechanisms may contribute to the cellular antiadhesive effects of phosphorothioate oligodeoxynucleotides. $\mathrm{Nu}$ cleic Acids Res. 24:737-745.

34. Bennett, M.R., and S.M. Schwartz. 1995. Antisense therapy for angioplasty restenosis: some critical considerations. Circulation. 92:1981-1993.

35. Lengyel, P. 1982. Biochemistry of interferons and their actions. Annu. 
Rev. Biochem. 51:251-282.

36. Hansson, G.K., L. Jonasson, J. Holm, M.M. Clowes, and A.W. Clowes. 1988. $\gamma$-Interferon regulates vascular smooth muscle cell proliferation and Ia expression in vivo and in vitro. Circ. Res. 63:712-719.

37. Hansson, G.K., M. Hellstrand, L. Rymo, L. Rubbia, and G. Gabbiani. 1989. Interferon- $\gamma$ inhibits both proliferation and expression of differentiationspecific $\alpha$-smooth muscle actin in arterial smooth muscle cells. J. Exp. Med. 170: $1595-1608$.

38. Hansson, G.K., and J. Holm. 1991. IFN- $\gamma$ inhibits arterial stenosis after injury. Circulation. 84:1266-1272.

39. Wang, W.Z., H.J. Chen, K.N. Giedd, A. Schwartz, P.J. Cannon, and L.E. Rabbani. 1995. T cell lymphokines, interleukin-4 and gamma interferon, modulate the induction of vascular smooth muscle cell tissue plasminogen activator and migration by serum and platelet-derived growth factor. Circ. Res. 77: 1095-1106.

40. Morishita, R., G.H. Gibbons, K.E. Ellison, M. Nakajima, L. Zhang, Y.
Kareda, T. Ogihara, and V.J. Dzau. 1993. Single intraluminal delivery of antisense $c d c 2$ kinase and proliferating-cell nuclear antigen oligonucleotides results in chronic inhibition of neointimal hyperplasia. Proc. Natl. Acad. Sci. USA 90:8474-8478

41. Morishita, R., G.H. Gibbons, K.E. Ellison, M. Nakajima, H. von der Leyen, L. Zhang, Y. Kareda, T. Ogihara, and V.J. Dzau. 1994. Intimal hyperplasia after vascular injury is inhibited by antisense $c d k 2$ kinase oligonucleotides. J. Clin. Invest. 93:1458-1464.

42. Abe, J., W. Zhou, J. Taguchi, N. Takuwa, K. Miki, H. Okazaki, K. Kurokawa, M. Kumada, and Y. Takuwa. 1994. Suppression of neointimal smooth muscle cell accumulation in vivo by antisense $c d c 2$ and $c d k 2$ oligonucleotides in rat carotid artery. Biochem. Biophys. Res. Commun. 198:16-24.

43. Simons, M., E.R. Edelman, and R.D. Rosenberg. 1994. Antisense proliferating-cell nuclear antigen oligonucleotides inhibit neointimal hyperplasia in a rat carotid artery injury model. J. Clin. Invest. 93:2351-2356. 